\title{
Magnetically Monitored Resonant Points of the Capacitance in Trilayer Composite TDF/PZT/FDF
}

\author{
Jiansen Gao ${ }^{1, ~ a}$, and Ning Zhang ${ }^{2, b}$ \\ ${ }^{1}$ Basic Teaching Department, Suqian College of Jiangsu Province, Suqian 223800, China \\ ${ }^{2}$ Physics Department, Nanjing Normal University, Nanjing 210023, China \\ ajsgao0408@163.com, bhangning@njnu.edu.cn
}

Keywords: Laminate composite, magnetoelectric coupling, magneto-capacitance effects

\begin{abstract}
Layered composite ferromagnet/piezoelectric /ferromagnet was fabricated. And its magneto-capacitance effect was studied. A few formants were discovered on the curves of frequency dependent capacitance. The formants were observed to shift with changing magnetic field, suggesting that resonant frequencies depend on the features of the magnetostriction of the ferromagnet. Giant magneto-capacitance effects were obtained near the resonant points under a magnetic field less than $1 \mathrm{kOe}$. Theoretical analysis showed that such magnetically tuned capacitance comes from the piezomagnetic effect of the ferromagnetic phase in the composite by means of the interfacial coupling.
\end{abstract}

\section{Introduction}

The magnetocapacitance (MC) is currently attracting much interest of study due to its potential applications in magnetic sensors, actuators and storage devices, etc [1-2]. Many studies have been reported on MC effect in the magnetoelectric materials since Suchtelen observed magnetoelectric effects in composites, [3]. Recently, there have been many investigations on the magnetic fieldtuned electro-mechanical resonances in magnetoelectric composites materials. [4-5]. Large MC effect can be expected near the formants of a laminate composite since any small change in frequency near resonance points will cause a dramatic change in the capacitance. In this study, we performed study on magnetic-field-tuned resonances in capacitance for layered composites.

\section{Sample Characterization}

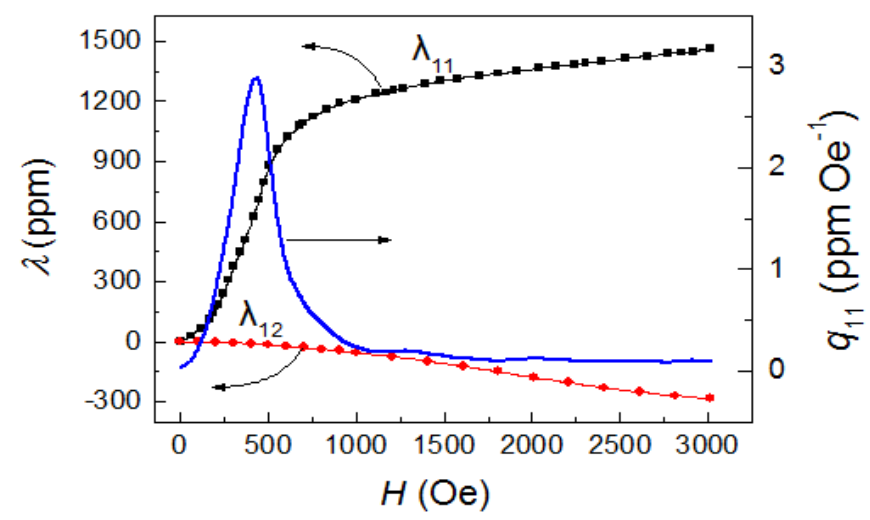

Figure 1 Applied magnetic field dependence of the magnetostriction and corresponding piezomagnetic coefficient for the Terfenol-D (TDF) used.

The sample investigated was a laminate of Terfenol-D/Pb ( $\mathrm{Zr}$, Ti)O3/Terfenol-D, where the $\mathrm{Pb}(\mathrm{Zr}, \mathrm{Ti}) \mathrm{O} 3$ (PZT) is the most often used piezoelectrics and Terfenol-D (TDF) is famous for its performance in magnetostriction. Commercial TDF and PZT plates were used in this work. The 
magnetostrictive $(\lambda)$ and the corresponding piezomagnetic $(\mathrm{q}=\mathrm{d} \lambda / \mathrm{dH})$ coefficients of the TDF were measured since they are important in this work. The results are shown in Figure 1.

Considering the TDF strip works under tight clamping condition, the measurement of it was measured by using a standard strain gage technique at a constant pressure of 8MPa. The length of the TDF strip was along the direction of the magnetic field in measurement. Figure 1 shows that, with increasing magnetic field $\mathrm{H}$, the magnetostrictive coefficient in length direction, $\lambda 11$, increases first rapidly when $\mathrm{H} \leq 750$ Oe, then slowly and approaches saturation at $\mathrm{H} \approx 3000$ Oe. And its saturated magnetostriction near $1470 \mathrm{ppm}$. The magnetostriction perpendicular to the magnetic field, $\lambda 12(13)$, was observed to be very small, thus, it can be ignored.

Both TDF and PZT plates were sliced in size of $20 \times 7 \times 2 \mathrm{~mm} 3$. The cut strips were bonded together by nonconductive epoxy adhesives to become a trilayer. For measurement the MC respond, the length of the laminate was set to be parallel to the field. An impedance analyzer (IM3570, Hioki E.E. Corporation) was used for measuring the capacitance along the thick direction of the PZT with an excitation voltage of $1 \mathrm{~V}$.

\section{Experimental Results And Discussion}

Figure 2 shows the frequency dependent capacitances under different magnetic fields. As can be seen, three formants are found in the scope of the frequencies manipulated at zero applied field. They located at $175.56 \mathrm{kHz}, 187.56 \mathrm{kHz}$ and $194.06 \mathrm{kHz}$, respectively. Huge variation of capacitance can be observed in the domains near the formants (fr). But the value displays relatively stable in other areas of frequency. Additionally, the formants vary obviously with increasing applied field, causing huge magnetic capacitance around the resonant points. For example, resonant frequency at the second peak decreases from fr=187.1 to $184.4 \mathrm{kHz}$ with increasing the field from 0 to 300 Oe,.

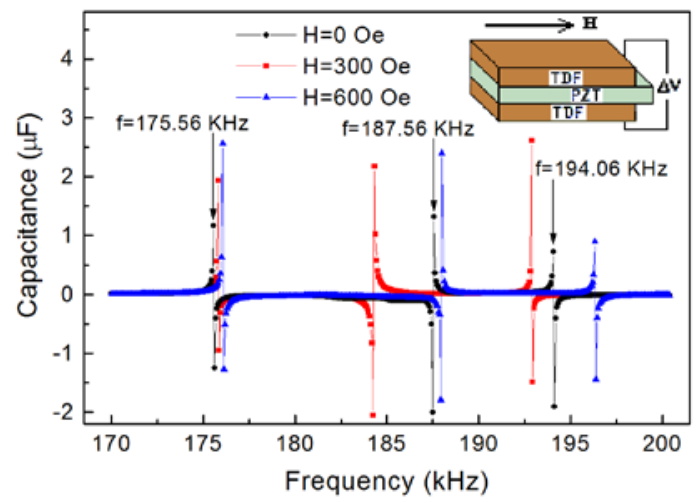

Figure 2 The frequency dependence of capacitance with and without magnetic field; Inset: the schematic program of the device investigated.

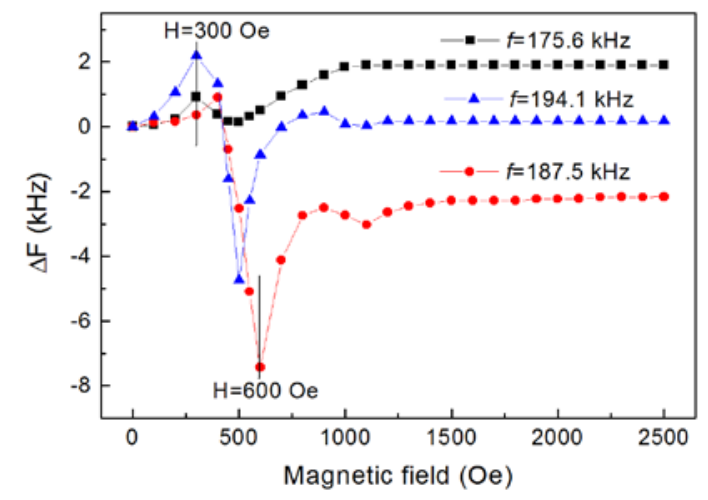

Figure 3 Magnetic field dependent frequency shifts at different resonant points of the sample.

To reveal the magneto-capacitance at the areas of resonance frequencies, field dependent shifts 
of frequency at different formants were investigated. Defined the frequency shift as $\Delta \mathrm{fr}=\mathrm{fH}$-f0, where $\mathrm{fH}$ and $\mathrm{f} 0$ are the frequencies under field and zero field, respectively. The measured results were shown in Figure 3. It is found that the shifts undergo a peak at first then a valley with increasing the field from zero to about 1500 Oe. Then all the shifts maintain stable at other frequencies.

As can be seen in Figure 4 with frequency shifting, great MC can be obtained near the formants. Additionally, it is found that the capacitances at different formants also experience a peak and a valley with increasing the field, suggesting that the impedance of the sample can be changed from capacitive type to inductive ones with changing the field. Thus, giant negative and positive magneto-capacitance effects can be observed simultaneously under a magnetic field less than $1 \mathrm{k}$ Oe near the formants.

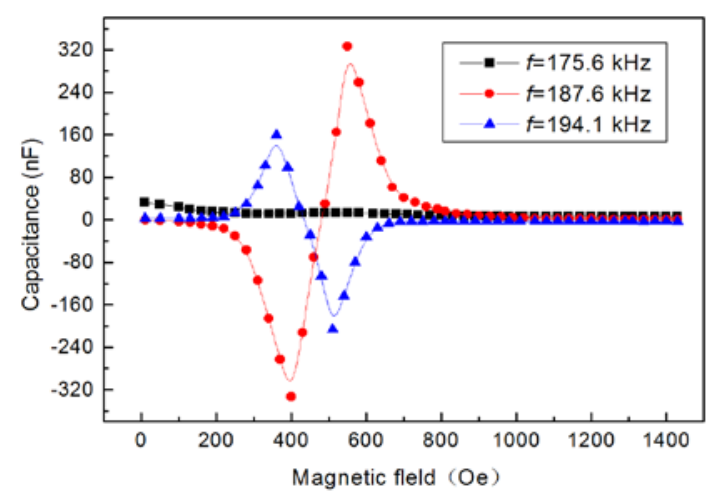

Figure 4 Magnetic field dependent capacitances at different resonant frequencies.

\section{Theoretical Analysis}

According to the theory of laminate composites [6], the sound speed in the present laminate can be expressed as

$$
\bar{v}=\sqrt{\frac{1}{\rho}\left(\frac{n}{{ }^{m} S_{11}}+\frac{1-n}{{ }^{E} S_{11}}\right)}
$$

Where $\rho$ is the average density of the composite, ${ }^{E} S_{11}$ and ${ }^{m} S_{11}$ are the compliances of PZT and Terfenol-D respectively, $n=2 \mathrm{tm} / \mathrm{tc}$, $\mathrm{tm}$ is the thickness of TDF, tc is the total thickness of the laminate. When under a magnetic field, ${ }^{m} s_{11}$ in Eq. (1) should be replaced with

$$
{ }^{m} S_{11}^{B}={ }^{m} S_{11}-{ }^{m} q_{11}^{2} / \mu_{11}
$$

Where ${ }^{m} q_{11}=\partial \lambda / \partial H$ is the piezomagnetic coefficient, $\mu 11$ magnetic permeability of Terfenol$\mathrm{D}$, and $\lambda$ is the magnetostrictive coefficient. Since ${ }^{m} q_{11}$ is generally positive, ${ }^{m} s_{11}^{B}$ should less than ${ }^{m} s_{11}$ under an applied field, leading to increase the sound speed in Terfenol-D with presence of a magnetic field. Therefore, magnetically tuned sound speed in the laminate composite can be achieved. On the other hand, the resonant frequency of longitudinal vibration mode of a piezoelectric is given by [7]

$$
f_{r}=\frac{k}{2 l} \bar{v}
$$

Where $k=0,1,2 \ldots, l$ is the length of the PZT. So we conclude that the magnetic field-tuned compliances of the ferromagnet are responsible for the variation of frequency in the laminate.

The capacitance with two plain plates is given by $C=\varepsilon_{33} A / d$, where $A$ is the area of a plate and $\mathrm{d}$ is the PZT thickness. Thus, according to the constitutive equations of the piezoelectric and 
magnetostrictive phases, and the interlayer elastic coupling [10], the expression of the capacitance for the composite can be written as

$$
\mathrm{C}=\frac{A}{d}\left[{ }^{p} \varepsilon_{33}+\frac{{ }^{p} d^{2}{ }_{31}}{{ }^{p} S_{11}}\left(\frac{f_{1}(H)}{f_{2}(H)} \frac{2 \tan \left[l \omega f_{2}(H) / 2\right]}{l \omega}-1\right)\right]
$$

Where $f_{1}(H)=\frac{v\left({ }^{m} s_{11}+{ }^{m} q_{11}^{2} / \mu_{11}\right)}{(1-v)^{p} s_{11}+v\left({ }^{m} s_{11}+{ }^{m} q_{11}^{2} / \mu_{11}\right)}, \quad f_{2}(H)=\sqrt{\left[\frac{v}{\left[{ }^{p} s_{11}\right.}+\frac{1-v}{{ }^{m} s_{11}+{ }^{m} q_{11}^{2} / \mu_{11}}\right]^{-1}}, \quad \omega=2 \pi f$ is angular frequency, ${ }^{p} \varepsilon_{33}$ and ${ }^{p} d_{31}$ are the permittivity and piezoelectric coefficient of PZT, respectively, $\bar{\rho}=v^{p} \rho+(1-v)^{m} \rho$ is the average mass density, $v$ is the PZT volume fraction, and ${ }^{\mathrm{p}} \rho$ and ${ }^{\mathrm{m}} \rho$ are the densities of PZT and Terfenol-D, respectively. In accordance with Eq. (3), the resonant condition should be

$$
\frac{l \omega_{r}}{2} f_{2}(H)=\left(\frac{1}{2}+\mathrm{k}\right) \pi
$$

Thus, the relation between resonant frequency and external magnetic field can be described as

$$
f_{r}(H)=\frac{1+2 k}{2 l \sqrt{\rho}} \sqrt{\frac{v}{{ }^{p} s_{11}}+\frac{\mu_{11}(1-v)}{{ }^{m} s_{11} \mu_{11}+q_{11}^{2}}}
$$

Using Eq. (6), the resonance points under zero field and the shifts of the formants with changing magnetic field can be worked out, respectively. The results are shown in Figure 5.

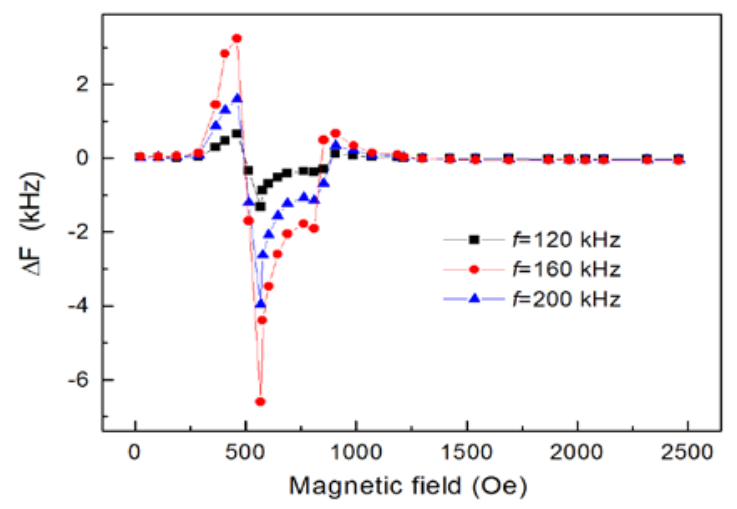

Figure 5 Calculated frequency shifts as functions of applied magnetic field.

The material parameters used in the calculation are ${ }^{\mathrm{m}_{\mathrm{s}}}{ }_{11}=12.5 \times 10^{-}$ ${ }^{11} \mathrm{~m}^{2} / \mathrm{N}, \quad{ }^{\mathrm{m}} \rho=9.2 \times 10^{3} \mathrm{~kg} / \mathrm{m}^{3}, \quad{ }_{\mathrm{p}} \mathrm{s}_{11}=15.3 \times 10^{-12} \mathrm{~m}^{2} / \mathrm{N}, \quad{ }^{\mathrm{p}} \mathrm{d}_{31}=-175 \times 10^{-12} \mathrm{~m} / \mathrm{N}, \quad{ }^{\mathrm{p}} \rho=7.5 \times 10^{3} \mathrm{~kg} / \mathrm{m}^{3}$, respectively [8-9], and the volume fraction of PZT is taken as $v=0.33$. As can be seen, the results shown in Figure 5 agree with that observed in experiments (in Figure 3), suggesting that the theoretical model discussed above is reasonable. According to Eqs. 4 and 6, both capacitance and resonant frequencies depend on the piezomagnetic coefficient of the ferromagnetic phase in the layered composite q11. Since the peak value of q11 is at magnetic field of 450 Oe, and the maximum values of the change rate of q11 with increasing magnetic field, (dq11/dH) max, locate at 300 Oe and 600 Oe, respectively, which corresponding to the rising and falling edges of the q11 peak, respectively, (Figure 1), we can see that both capacitance and resonant frequencies show greatest change near the two values of magnetic field, thus leading to giant magneto-capacitance.

\section{Conclusion}

The features of the longitudinal piezomagnetic coefficient with changing magnetic field for a given ferromagnet depend on its magnetostrictive performance in the same direction, and lead to resonant of magnetocapacitive effect in the layered composites based on the magnetostriction of the magnet. Since the resonant frequencies also depend on the piezomagnetic coefficients in the 
composites, one can observe the magnetical shift of resonant points, and the resulting giant negative and positive magneto-capacitance effects around the resonant frequencies.

\section{References}

[1] M. A. Subramanian, T. He, J. Z. Chen, N. S. Rogado, T. G. Calvarese, and A. W. Sleight, "Giant Room-Temperature Magnetodielectric Response in the Electronic Ferroelectric LuFe2O4" Adv. Mater., vol. 18, pp. 1737-1739, May 2006.

[2] H. Kaiju, S. Fujita, T. Morozumi, and K. Shiiki, "Magnetocapacitance Effect of Spin Tunneling Junctions” J. Appl. Phys., vol. 91, pp. 7430-7435, December 2002.

[3] J. V. Suchtelen, "Product properties of a new application of composite materials" Philips Res. Rep., vol. 27, pp. 28-37, January 1972.

[4] Y. Wang, H. Yu, M. Zeng, J. G. Wan, M. F. Zhang, J.-M. Liu, and C. W. Nan, "Numerical modeling of the magnetoelectric effect in magnetostrictive piezoelectric bilayers" Appl. Phys. A: Mater. Sci. Process. vol. 81, pp. 1197-1202, 2005.

[5] C. Israel, V. M. Petrov, G. Srinivasan, and N. D. Mathur, "Magnetically tuned mechanical resonances in magnetoelectric multilayer capacitors” Appl. Phys. Lett. vol. 95, pp. 072505, August 2009.

[6] Shuxiang Dong, Jie-Fang Li, and Dwight Viehland, "Longitudinal and transverse magnetoelectric voltage coefficients of magnetostrictive /piezoelectric laminate composite: theory" IEEE Transactions on Ultrasonics, Ferroelectrics, and Frequency Control., vol. 50, pp. 1253-1261, November 2003.

[7] J. G. Wan, Z. Y. Li, Y. Wang, M. Zeng, G. H. Wang, and J.-M. Liu, "Strong flexural resonant magnetoelectric effect in Terfenol-D/epoxy-Pb(Zr, Ti)O3 bilayer” Appl. Phys. Lett., vol. 86, pp. 202504, May 2005.

[8] A Bayrashev,W P Robbins and B Ziaie, "Low frequency wireless powering of microsystems using piezoelectric-magnetostrictive laminate composites" Sensors and Actuators A, vol. 114, pp. 244-249, September 2004.

[9] M I Bichurin,V M Petrov and G Srinivasan, "Theory of low-frequency magnetoelectric coupling in magnetostrictive-piezoelectric bilayers” Phys. Rev. B, vol. 68, pp. 0544021-05440213, April 2003. 